\title{
Importancia de la velocidad de onda de corte y del período predominante para la evaluación de la respuesta de sitio en Santiago
}

The importance of the shear wave velocity and the predominant period for the evaluation of the site response in Santiago

Fecha de entrega: 16 de diciembre 2014 Fecha de aceptación: 6 de abril 2015

\section{Cristian Godoy ${ }^{1}$, Lenart González ${ }^{2}$ y Esteban Sáez}

${ }^{1}$ SRK Consulting, Vitacura 2939, piso 5, Las Condes, Santiago, Chile, cgodoy@srk.cl

${ }^{2}$ IDIEM, Universidad de Chile, Plaza Ercilla 883, Santiago, Chile, lenart.gonzalez@idiem.cl

${ }^{3}$ Departamento de Ingeniería Estructural y Geotécnica, P. Universidad Católica de Chile, Vicuña Mackenna 4860, Macul, Santiago, Chile, esaez@ing.puc.cl

Este artículo presenta los resultados del estudio de respuesta sísmica en diferentes sitios de Santiago realizado con el método lineal-equivalente. Para lo anterior, se desarrolló una metodología que permite establecer perfiles "equivalentes" de $V_{s}$ hasta el contraste de impedancia predominante de profundidad mayor a $100 \mathrm{~m}$, utilizando mediciones superficiales de $V_{s} y$ de periodo predominante mediante la técnica de Nakamura o HVR. Una vez obtenidos los perfiles a analizar, se comparó la respuesta modelada con la real, obtenida a partir de registros sísmicos de eventos de $M_{w}$ variable entre 4.0 y 8.8 , obteniéndose una representación adecuada. Finalmente, se realizó un estudio paramétrico de respuesta de sitio considerando la variación de la profundidad y la estratigrafía de la cuenca de Santiago. Los resultados evidencian la importancia de utilizar el periodo predominante en conjunto con el $V_{s}$ para una caracterización dinámica adecuada de los sitios estudiados.

Palabras clave: velocidad de onda de corte, periodo predominante, respuesta desitio, método lineal-equivalente
This article presents the results of a seismic response study of different sites in Santiago conducted with the equivalent linear method. A methodology was developed to determine "equivalent" profiles of $V_{s}$ up to the predominant impedance contrast (more than $100 \mathrm{~m}$ of depth), using shallow measurements of $V_{s}$ and the predominant period through the Nakamura's technique or HVR. Once the profiles to be analyzed were obtained, the real and the modelled responses were compared for seismic events with $M_{w}$ ranging from 4.0 to 8.8 , yielding to an adequate representation. Finally, a parametric analysis of site response was performed considering the variation of the depth and stratigraphy across the Santiago basin. The trends of the seismic response evidence the importance of using the predominant period alongside the $V_{s}$ for an adequate dynamic characterization of the studied sites.

Keywords: shear wave velocity, predominant period, site response, equivalent-linear method

\section{Introducción}

El terremoto del 27 de febrero del año 2010 es considerado uno de los más grandes eventos sísmicos de la historia. $\mathrm{Su}$ efecto ocasionó grandes daños que se extendieron desde la región de la Araucanía hasta la de Valparaíso. Esto trajo consigo la necesidad de reevaluar ciertos aspectos de la práctica nacional para mejorar y optimizar los diseños de ingeniería. En este ámbito se generó una discusión sobre la normativa vigente de diseño sísmico de estructuras y en particular sobre el sistema de clasificación sísmica de suelos (Norma NCh 433 of. 1996 mod. 2009). Lo expresado anteriormente motivó la realización de una investigación tendiente a mejorar el conocimiento de la respuesta de sitio, en este caso, en la cuenca de Santiago. 
Específicamente, se analizaron los parámetros que pueden influir en la respuesta de sitio y que podrían ser utilizados para mejorar el sistema de clasificación actual. Para lo anterior, se analizó el comportamiento de distintos sitios ubicados en la cuenca de Santiago utilizando el método lineal-equivalente, para determinar si el parámetro de clasificación actual (velocidad de onda de corte promedio en los primeros $30 \mathrm{~m}$ o $V_{s 30}$ ) es adecuado y suficiente para modelar la respuesta en superficie, y de lo contrario complementarlo con, por ejemplo, el período predominante $T_{0}$ del sitio para mejorar las predicciones.

\section{Metodología}

La metodología seguida consistió en la selección de los sitios a estudiar, registros sísmicos a utilizar, determinación de perfiles unidimensionales de velocidad de onda de corte $V_{\text {s }}$ para los sitios escogidos, verificación de la capacidad de predicción del método lineal-equivalente y el análisis de respuesta sísmica para distintos sitios en la cuenca de Santiago, identificando tendencias para los parámetros de clasificación de sitio estudiados.

\section{Selección de sitios}

El criterio para la selección de sitios dentro de la cuenca de Santiago corresponde a la presencia de una estación de monitoreo acelerográfico y contar con, al menos, una medición de $V_{\mathrm{s}}$ cercana. Si bien disponer de mediciones de período predominante y descripciones estratigráficas era deseable, estos factores no fueron considerados como determinantes para la elección. Con este criterio en vista, los sitios seleccionados correspondieron a las estaciones de Santiago Centro, Maipú y Puente Alto, pertenecientes a RENADIC; Quilicura, Pudahuel y La Reina, pertenecientes a la red SMASCH de la PUC; y finalmente la estación Antumapu, correspondiente al Servicio Sismológico Nacional SSN. La ubicación de las estaciones se presenta en la Figura 1. Estas estaciones se distribuyen a través de toda la zona urbana de la cuenca de Santiago y se sitúan sobre las distintas unidades geológicas presentes en ella. El detalle de la información utilizada se puede encontrar en Godoy (2013).

Los registros en la estaciones en roca y en los sitios de estudio fueron aportados por Universidad de Chile
(RENADIC), P. Universidad Católica (red SMASCH), Servicio Sismológico Nacional y SOCHIGE.
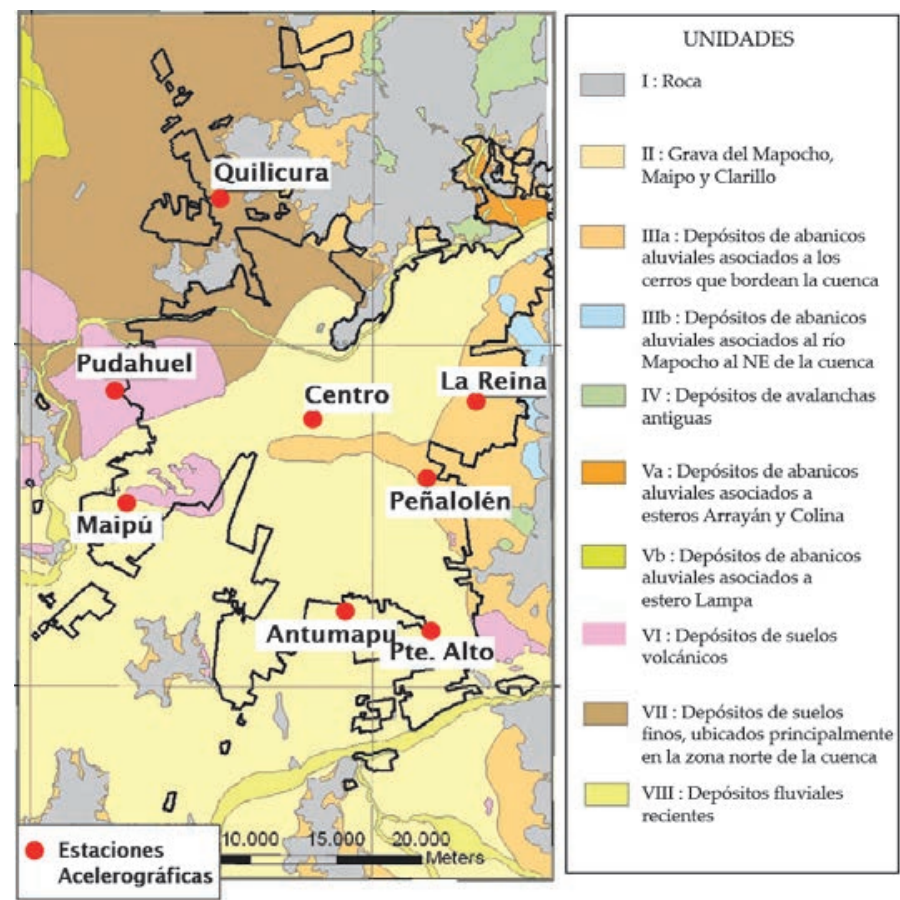

Figura 1: Estaciones acelerográficas seleccionadas y mapa de depósitos de suelos de Santiago (Leyton et al., 2011)

\section{Selección de registros sísmicos}

Los registros sísmicos seleccionados para el estudio se pueden agrupar en dos casos:

Caso I: corresponde a distintos registros del terremoto del 27 de febrero del $2010\left(M_{w}=8.8\right)$, obtenidos en distintas estaciones acelerográficas ubicadas en roca. La respuesta en suelo, en este caso, es medida por las estaciones Antumapu, Maipú, Puente Alto, Peñalolén y Santiago Centro.

Caso II: corresponde a distintos eventos de baja a mediana magnitud (variable entre 4.5 y 6.9 ) registrados en la estación Cerro Santa Lucía (del SSN) ubicada en roca. La respuesta en suelo, para este caso, fue medida en las estaciones Quilicura, La Reina, Pudahuel y Antumapu.

Las Figuras 2 y 3 muestran la distribución espacial de las estaciones donde fueron registrados los eventos en cada caso, así como los gráficos tiempo-historia de cada uno de los registros. 


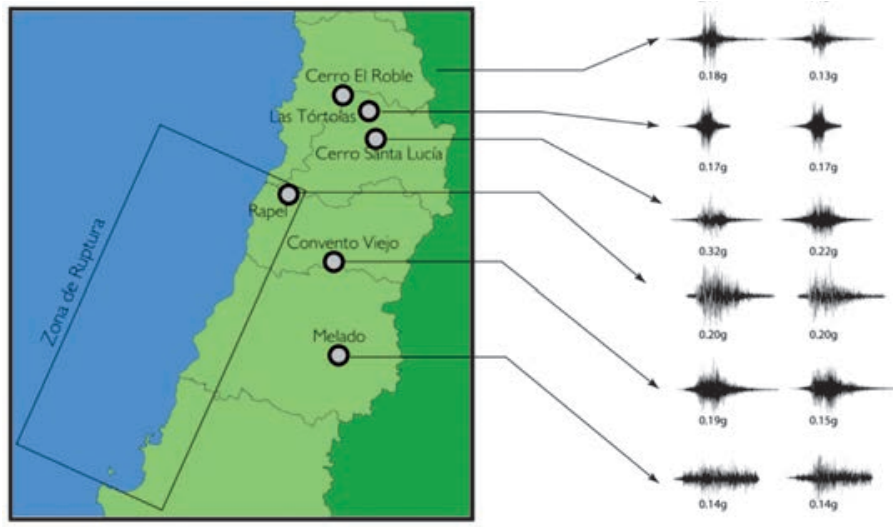

Figura 2: Registros y ubicación de estaciones acelerográficas en roca para el caso I

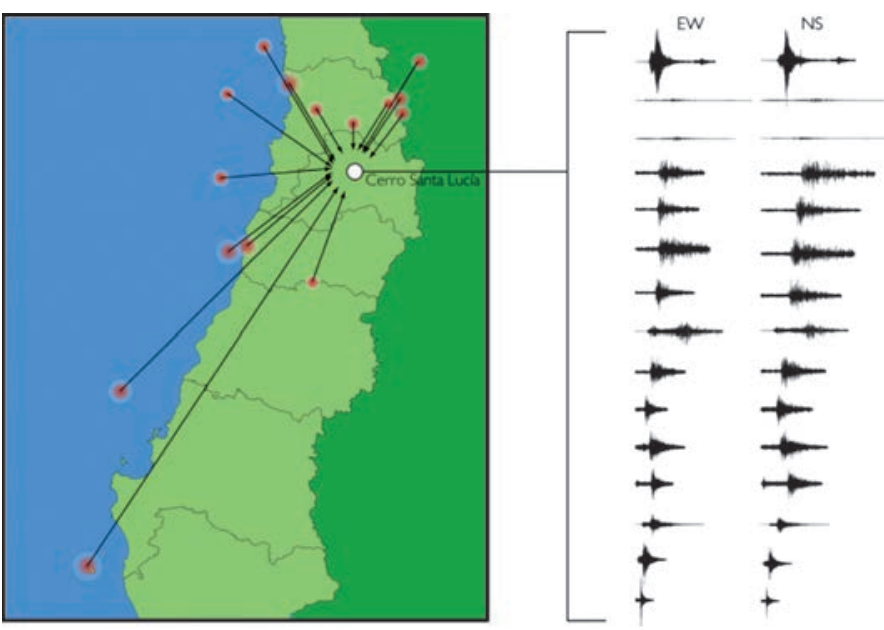

Figura 3: Registros y ubicación de la estación acelerográfica en roca para caso II

\section{Determinación de perfiles unidimensionales}

Si bien el requisito de selección de sitios consistió en disponer de mediciones in situ de $V_{\mathrm{s}}$, estos datos no son suficientes para caracterizar el perfil completo de suelo hasta la roca. Por lo anterior, se debió recurrir a una metodología alternativa que permitiera extender los perfiles de $V_{\mathrm{s}}$ hasta el contraste de impedancia predominante o roca "aparente". La metodología consiste en determinar un perfil "equivalente" a través de una calibración con el período predominante del suelo, la profundidad al basamento y la estratigrafía predominante de la zona, según se presenta en la Figura 4. En los sitios que no se disponía de información del período $T_{o} \mathrm{y} / \mathrm{o}$ de estratigrafías, estos parámetros fueron interpolados de puntos de mediciones cercanos (Godoy, 2013).
Los datos de profundidad del basamento rocoso fueron obtenidos a partir de mediciones gravimétricas de Araneda et al. (2000). Lo anterior es utilizado bajo la hipótesis de que el período predominante corresponde para estos casos al fundamental, de forma que el contraste de impedancia que define el periodo de vibrar predominante del sitio coincide con el período hasta la roca. Bajo esta hipótesis, es adecuado utilizar para la calibración la combinación del período predominante con la profundidad gravimétrica. Como se mencionó anteriormente, el perfil "equivalente" corresponde a aquél que mejor interpreta el período predominante, es decir, el período de vibración del sitio a bajas deformaciones. Por lo tanto, es correcto determinar esta respuesta a través de un análisis lineal-elástico del suelo. Estos análisis se realizaron sobre perfiles de prueba, los cuales son obtenidos completando el perfil de $V_{s}$ para las profundidades donde no se conoce, asumiendo un crecimiento de $V_{\mathrm{s}}$ con la profundidad, según la siguiente expresión:

$V_{\mathrm{s}}(z)=k z^{\alpha}$

donde $k$ es una constante de ajuste, $z$ es la profundidad y $\alpha$ varía entre 0 y 0.25 dependiendo del tipo de material. $V_{\mathrm{s}}$ del basamento rocoso ha sido asumido constante e igual a $2000 \mathrm{~m} / \mathrm{s}$. De esta forma, se generan diferentes perfiles que entregan diferentes respuestas en bajas deformaciones $y$, por ende diferentes períodos predominantes. El perfil equivalente para el sitio en estudio corresponde a aquél con el $T_{0}$ más cercano al medido o estimado para éste. Lo anterior se muestra gráficamente en la Figura 5.

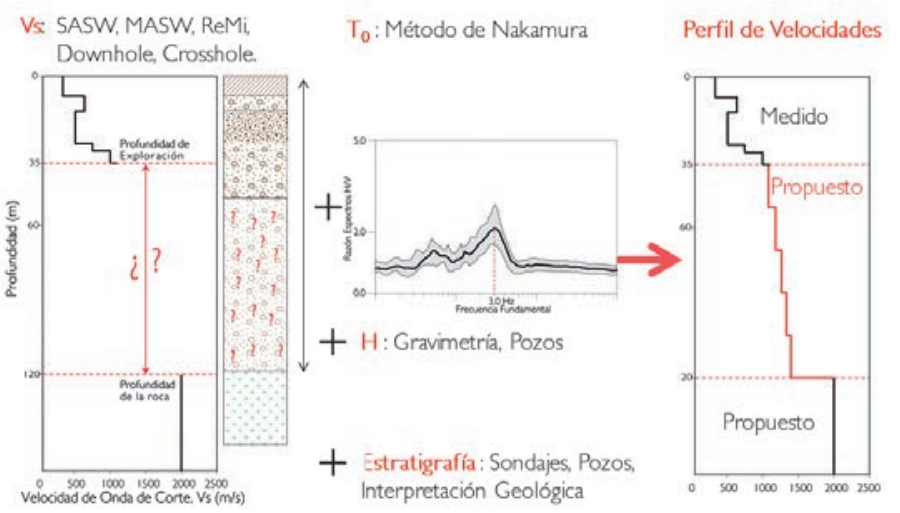

Figura 4: Metodología para la elaboración de perfiles de $V_{\mathrm{s}}$ equivalentes 


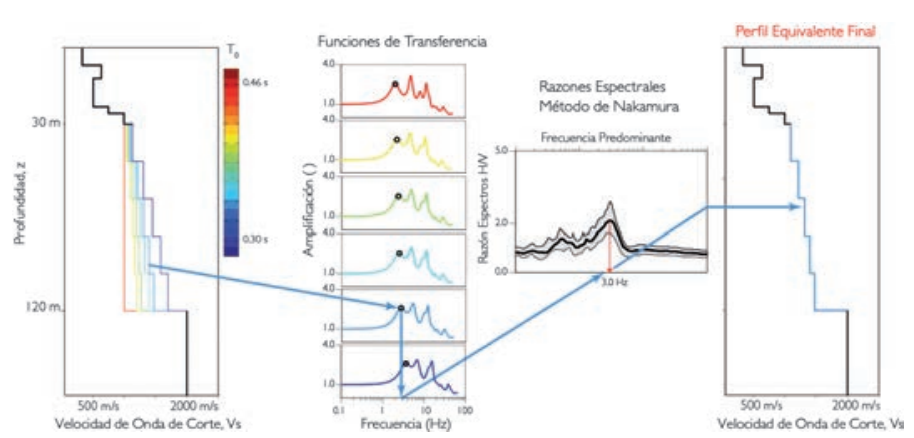

Figura 5: Ejemplo de elaboración de perfiles de prueba y selección del perfil equivalente final

En los sitios Peñalolén y Santiago Centro, el espectro de Nakamura se presentó con forma plana, no pudiéndose identificar claramente un período predominante. Para estos casos, y para no desperdiciar la información proveniente de estos perfiles, se asumieron tres períodos predominantes para cada uno, los cuales serán evaluados si corresponden o no más adelante. La Tabla 1 resume la información relevante de cada uno de los casos considerados.

Tabla 1: Resumen de propiedades de los perfiles analizados

\begin{tabular}{|l|c|c|c|c|}
\hline \multirow{2}{*}{ Perfil } & $T_{0}, \mathrm{~s}$ & $V_{\mathrm{s}}, \mathrm{m} / \mathrm{s}$ & $\begin{array}{c}V_{\mathrm{s}} 30, \\
\mathrm{~m} / \mathrm{s}\end{array}$ & $H, \mathrm{~m}$ \\
\hline \multirow{3}{*}{ Peñalolén } & $0.8^{*}$ & $663^{*}$ & \multirow{3}{*}{415} & 170 \\
\cline { 2 - 3 } & $1.0^{*}$ & $590^{*}$ & 415 & 170 \\
\cline { 2 - 3 } & $1.3^{*}$ & $605^{*}$ & & $220^{* *}$ \\
\hline Antumapu & 1.0 & $744^{*}$ & 428 & 220 \\
\hline Puente Alto & 1.0 & $847^{*}$ & 480 & 145 \\
\hline \multirow{3}{*}{ Santiago Centro } & $0.1^{*}$ & $1112^{*}$ & \multirow{3}{*}{641} & 100 \\
\cline { 2 - 3 } \cline { 5 - 5 } & $0.3^{*}$ & $981^{*}$ & 100 \\
\cline { 2 - 3 } & $0.5^{*}$ & $905^{*}$ & & $130^{* *}$ \\
\hline Maipú & 0.4 & $890^{*}$ & 478 & 120 \\
\hline Quilicura & 1.0 & $445^{*}$ & 360 & 125 \\
\hline La Reina & 1.8 & $776^{*}$ & 446 & 400 \\
\hline Pudahuel & 2.1 & $714^{*}$ & 523 & 400 \\
\hline
\end{tabular}

* Dato estimado, el resto proviene de mediciones reales o interpolación de mediciones reales. $V_{\mathrm{s}}$ y $V_{\mathrm{s} 30}$ corresponden a los $V_{\mathrm{s}}$ promediados (media armónica) en todo el depósito de suelo y hasta los primeros 30 $\mathrm{m}$, respectivamente. $H$ corresponde a la profundidad aproximada del basamento, obtenida de interpolación de las curvas de gravimetrías de Araneda et al. (2000).

** Estas profundidades fueron ajustadas para que el perfil entregara el $\mathrm{T}_{0}$ correspondiente.

Finalmente, con base en la información estratigráfica asociada a cada sitio, se asigna a cada tipo de material su correspondiente curva de degradación de módulo de corte, curva de amortiguamiento en función de la deformación angular y densidad. Lo anterior se realiza con valores obtenidos de la literatura (Aghaei Araei et al., 2010; Seed e Idriss, 1970; Vucetic y Dobry, 1991).

\section{Verificación del método lineal-equivalente}

Una vez que se elaboraron los perfiles unidimensionales, se determinó la respuesta a través del método linealequivalente, utilizando los sismos de entrada mencionados anteriormente. La respuesta obtenida de esta forma fue comparada con la respuesta real medida en cada sitio a través del coeficiente de eficiencia de Nash y Suttcliffe (1970), tanto de forma individual para cada sismo, así como la eficiencia promedio del grupo de sismos utilizados. Para las comparaciones antes descritas, se utilizó el espectro de respuesta, debido a que incorpora información de amplitud, duración y contenido de frecuencias del sismo en una sola curva. La Tabla 2 muestra los valores máximos, mínimos y promedio (mediana) de las eficiencias de cada grupo para el Caso I. El coeficiente de eficiencia puede variar entre $-\infty$ y $100 \%$, en general, eficiencias sobre $0 \%$ se consideran aceptables (Moriasi et al., 2007). La Figura 6 muestra los espectros de respuesta obtenidos. Es posible observar un muy buen ajuste en la mayoría de los casos, a excepción del sitio de Santiago Centro, donde si bien existe un espectro de respuesta muy similar al real, el comportamiento del grupo se aleja bastante de las mediciones para los tres períodos seleccionados.

Tabla 2: Coeficientes de eficiencia de Nash-Suttcliffe. Caso I.

\begin{tabular}{|l|c|c|c|}
\hline Estación & Mínima, \% & Máxima, \% & Mediana, \% \\
\hline Peñalolén & -26 & 79 & 24 \\
\hline Antumapu & -53 & 79 & 45 \\
\hline Puente Alto & 23 & 82 & 57 \\
\hline Maipú & 2 & 87 & 50 \\
\hline Santiago Centro & -441 & 68 & 10 \\
\hline
\end{tabular}

Para el caso de Peñalolén, el perfil estimado que entregó mejor ajuste fue aquél con un período predominante de 0.8 $\mathrm{s}$, por otra parte para la estación Santiago Centro fue el de 0.3 s. Sin embargo, como se comentó anteriormente, el segundo caso presenta grandes deficiencias en cuanto al ajuste para el grupo de sismos seleccionados, lo cual puede deberse al bajo contraste de impedancias del sitio, dificultando su caracterización a través de un período predominante. 

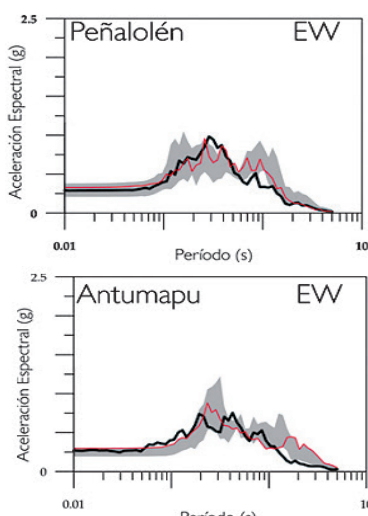

$0.01 \quad$ Periodo (s)
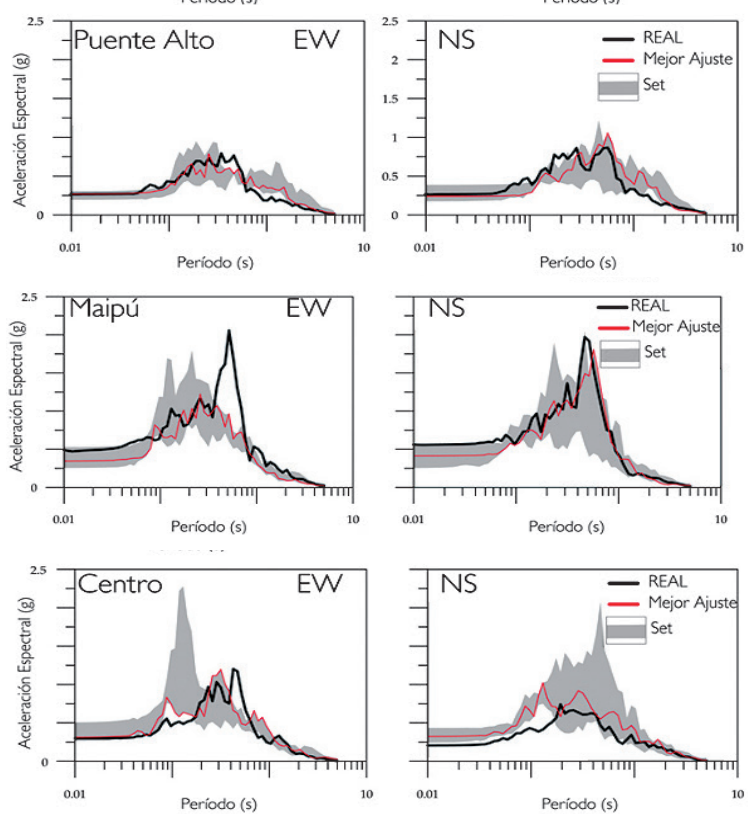

Figura 6: Espectros de respuesta modelados y reales para las estaciones estudiadas. Caso I

La Figura 7 presenta los resultados de las eficiencias para las distintas estaciones en suelo del Caso II. Es posible apreciar que los resultados arrojan muy buenos ajustes entre la modelación lineal-equivalente y la respuesta real, obteniéndose un $40 \%$ de los casos evaluados con eficiencias sobre $50 \%$, y $70 \%$ de los casos sobre $0 \%$.

Con respecto a estos resultados, es posible comentar que considerando las incertidumbres asociadas, tanto a los perfiles como a los sismos de entrada, los cuales están relacionados a estaciones distantes de los sitios estudiados, y que además, no existió calibración de las propiedades del suelo; el método lineal-equivalente es capaz de interpretar, en gran parte de los casos, de manera adecuada la respuesta en superficie. Sin embargo, es muy importante contar con una buena caracterización del sitio de estudio, principalmente en cuanto a la determinación del $V_{\mathrm{s}}$ y $T_{0}$.

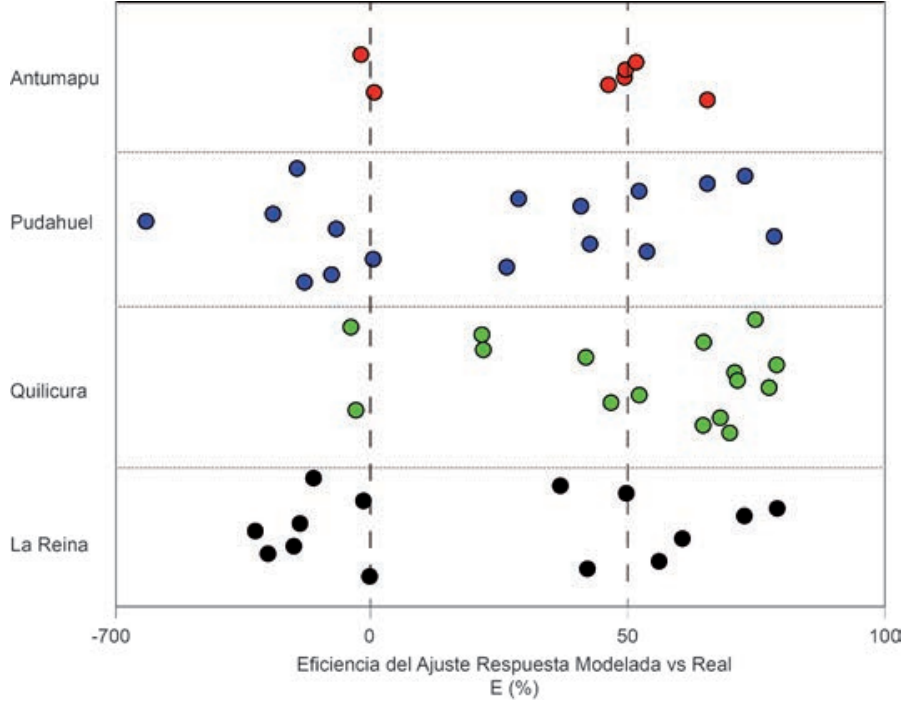

Figura 7: Eficiencia del ajuste caso II

\section{Análisis paramétrico para la cuenca de Santiago}

Finalmente, se realizó un análisis de respuesta de sitio paramétrico, sobre la base de los perfiles seleccionados y asumiéndolos representativos del sector en el que se ubican. La metodología consistió en mantener los primeros $50 \mathrm{~m}$ de cada perfil constante y a partir de eso, generar diferentes perfiles, de profundidad hasta el basamento variable entre $50 \mathrm{~m}$ y $500 \mathrm{~m}$, obteniéndose así perfiles con distinto $V_{\mathrm{s}}$ promedio (idéntico $V_{\mathrm{s} 30}$ ) y distinto $T_{0}$. La Figura 8 esquematiza lo mencionado anteriormente.

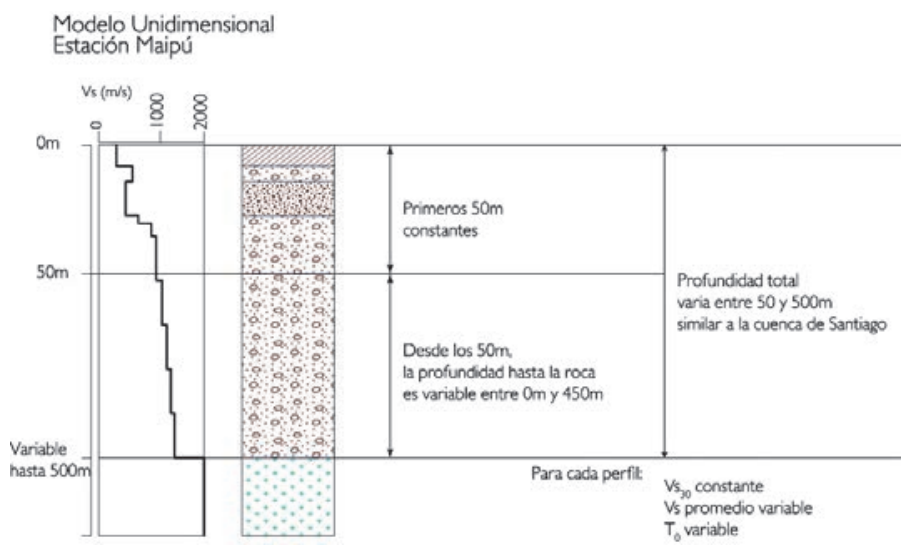

Figura 8: Esquema de la metodología de generación de perfiles generalizados para Santiago

Posteriormente, se procedió a realizar un análisis de respuesta de sitio a través del método lineal-equivalente para cada uno de estos perfiles y se identificaron las tendencias con distintos indicadores de la severidad de 
la respuesta (aceleración espectral máxima, intensidad de Arias, factor de amplificación promedio); de esta forma se obtuvo una correlación clara entre el $V_{\mathrm{s} 30}$ en conjunto con el período predominante y estos indicadores. Esta correlación no pudo ser conseguida utilizando sólo $V_{\mathrm{s} 30} \mathrm{O}$ $T_{\mathrm{o}}$ por separado, tal como se muestra en la Figura 9. Los resultados encontrados en esta etapa indican la importancia para la clasificación sísmica de sitio de la incorporación de un parámetro que dé cuenta de las características dinámicas de todo el perfil como el período predominante y no tan solo de los $30 \mathrm{~m}$ superiores. La Figura 10 esquematiza un ejemplo de cómo esto podría ser incorporado en las normativas, a partir de la tendencia observada en la Figura 9.

a)

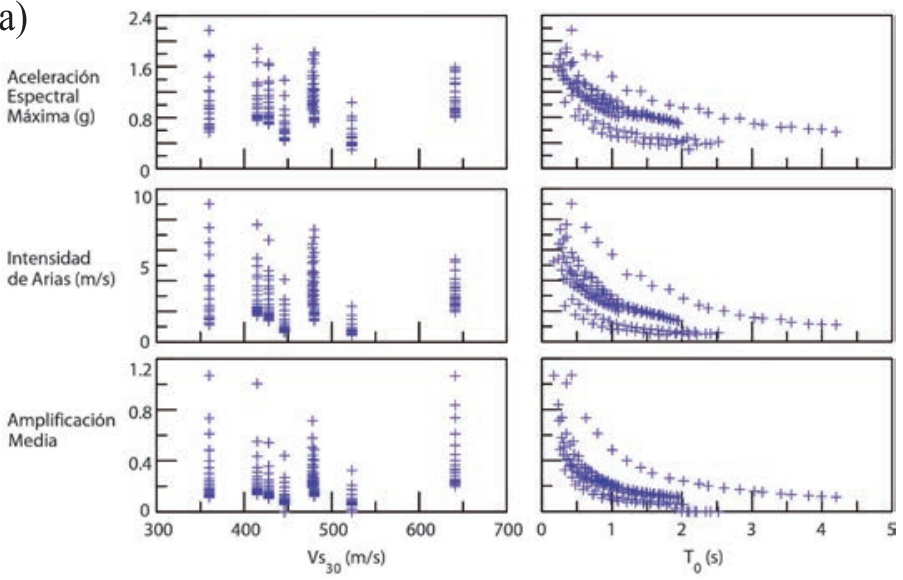

b)

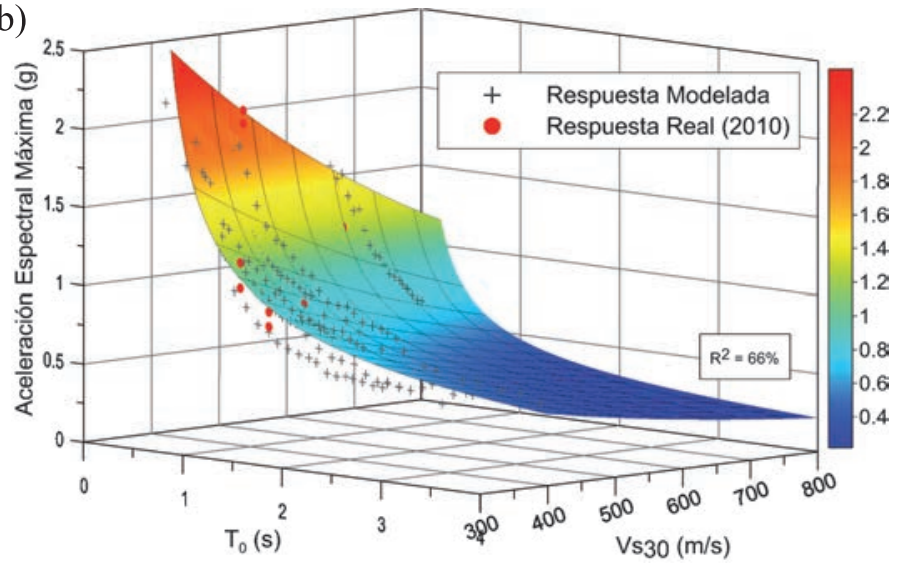

Figura 9: Tendencia de la respuesta de sitio utilizando $V_{\mathrm{s} 30}$ y $T_{0}$ a) por separado y b) juntos

\section{Conclusiones}

El presente trabajo demuestra la correlación que existe entre la respuesta sísmica de un sitio y el conjunto de parámetros formado por el $V_{\mathrm{s} 30} \mathrm{y}$ el período predominante. A partir de lo anterior, se destaca la importancia que tiene incluir el

\section{Clasificación DS N61}

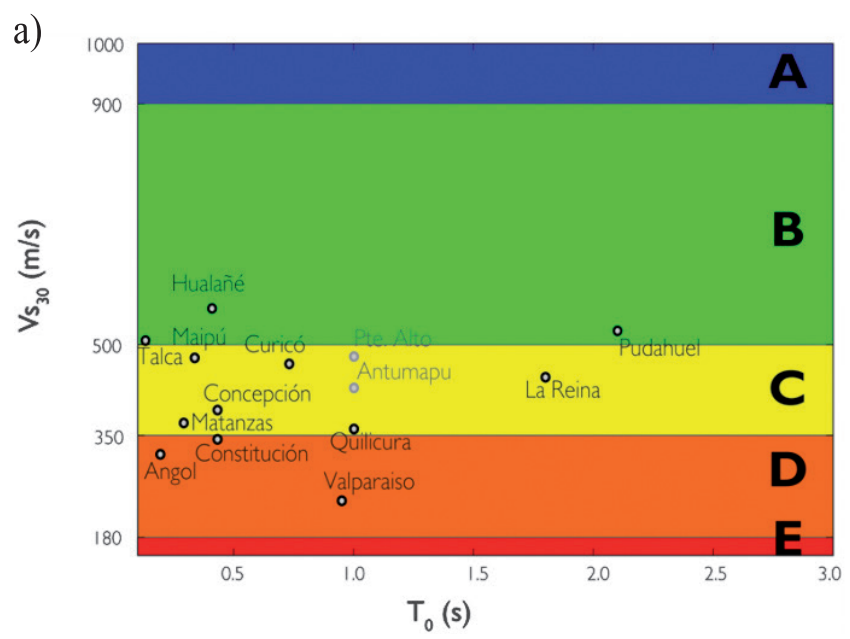

Clasificación considerando $\mathrm{Vs}_{30}+\mathrm{T}_{0}$

b)

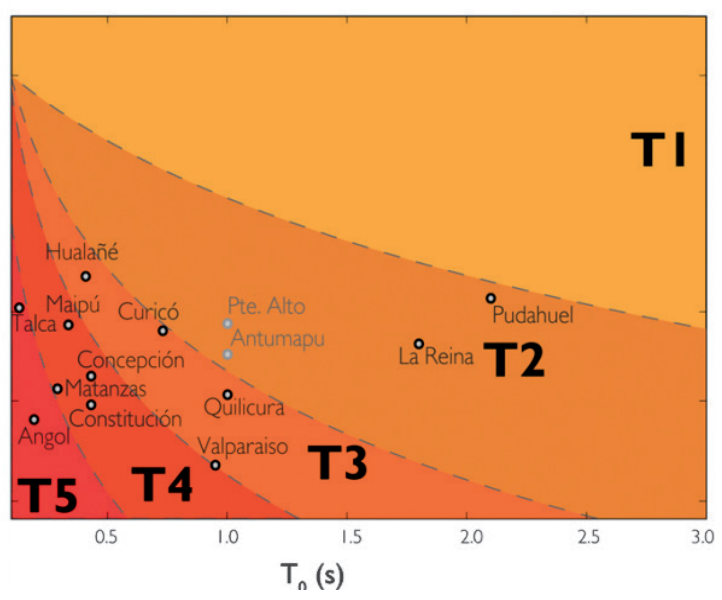

Figura 10: a) Sistema de clasificación actual (DS Nº61, 2011) y b) ejemplo de clasificación utilizando $V_{\mathrm{s} 30}$ y $T_{0}$

período en futuros sistemas de clasificación sísmica de suelos. También fue posible analizar adecuadamente la respuesta sísmica en la cuenca de Santiago a través del método lineal-equivalente. Esto podría ser generalizado a otras cuencas de similares características, es decir, formadas por suelos relativamente rígidos y con nivel freático profundo (o bien, con generación de presiones de poros despreciable).

Adicionalmente, se entregó una metodología para estimar un perfil "equivalente" de $V_{\text {s }}$ para depósitos más profundos donde no sea posible estimar la velocidad de ondas de corte hasta la roca basal. Se destaca la importancia de una buena caracterización de sitio para la realización de este tipo de estudios, en particular, definir el periodo predominante de manera adecuada. Todo lo anterior puede ser implementado 
como herramienta para estudios de microzonificación sísmica y estudios de riesgo sísmico locales.

Finalmente, cabe destacar, que en el proceso de calibración de los perfiles equivalentes, se asumió que el período predominante coincide con el período fundamental, aceptando que es posible utilizar la gravimetría para definir la profundidad al basamento. Sin embargo, y a pesar de que la mayoría de los perfiles calibrados entregaron resultados satisfactorios, pueden existir casos en que esta hipótesis entregue resultados inadecuados. Del mismo modo, la velocidad de onda de corte de la roca, que en este trabajo fue asumida constante e igual a $2000 \mathrm{~m} / \mathrm{s}$, puede tener influencia en los resultados de la calibración y posteriores análisis de respuesta de sitio.

\section{Referencias}

Aghaei Araei, A., Razeghi, H.R. and Tabatabaei, S.H. (2010). Dynamic properties of gravelly materials. Sciencia Iranica, Transaction A: Civil Engineering 17(4), 245-261

Araneda, M., Avendaño, M. y Merlo, C. (2000). Modelo gravimétrico de la cuenca de Santiago, etapa III final. IX Congreso Geológico de Chile, Puerto Varas, 2, 404-408

DS 61 (2011). Decreto Supremo $N^{\circ} 61$. Diario Oficial de la República. Cuerpo I-8. Martes 13 de Diciembre de 2011. MINVU
Godoy, C. (2013). Estudio de la respuesta de sitio en Santiago mediante el método lineal equivalente. Tesis MSc, Universidad de Chile

Leyton, F., Sepúlveda, S., Astroza, M., Acevedo, P., Ruiz, S., González, L. and Foncea, C. (2011). Seismic zonation of the Santiago Basin, Chile. 5th International Conference on Earthquake Geotechnical Engineering, Santiago, Chile

Moriasi, D.N., Arnold, J.G., Van Liew, M.W., Bingner, R.L., Harmel, R.D. and Veith, T.L. (2007). Model evaluation guidelines for systematic quantification of accuracy in watershed simulations. Transactions of the Asabe 50, 885-900

Nash, J.E. and Sutcliffe, J.V. (1970). River flow forecasting through conceptual models; Part I, a discussion of principles. Journal of Hydrology 10, 282-290

NCh 433 of. 1996 (2009). Diseño sísmico de edificios. Instituto Nacional de Normalización

Seed, H.B. and Idriss, I.M. (1970). Soil moduli and damping factors for dynamic response analyses. EERC, University of Berkeley

Vucetic, M. and Dobry, R. (1991). Effect of soil plasticity on cyclic response. Journal of Geotechnical Engineering, 117(1), 89-107 